\title{
Moving Object Tracking Based on Gaussian Kernel and Template Modelling
}

\author{
R. Raj Bharath, D. Bavya and P. Bavani, \\ Assistant Proffessor,Department of CSE \\ Manakula Vinayagar Institute of Technology \\ Puducherry, India, \\ PG Scholar, Department of CSE \\ Manakula Vinayagar Institute of Technology \\ Puducherry, India, \\ PG Scholar, Department of CSE \\ Manakula Vinayagar Institute of Technology \\ Puducherry, India. \\ rajbharathraj@gmail.com,d.bavya91@gmail.com,bavanichla77@gmail.com
}

\begin{abstract}
The Project presents object tracking from videos based on template matching using Gaussian kernel with probability distribution function and mean shift algorithm. Initially, the target will be selected from chosen video sequence to track desired object in consecutive frames. The target will be utilized to determine the probability distribution function for similarity measurement between target and current processing frames. Here, Gaussian kernel function and its gradient are used here to find the PDF for corresponding templates. Similarity between two different images will be measured by weighted sum of Gaussian coefficients and PDFS. Mean shift approach used here to shifting the starting coordinates of template to find its similar features in consecutive frames to detect desired objects. The dissimilarity between the target model and target candidates will be expressed by a metric derived from Bhattacharyya coefficient. The project simulated results shows that moving object from video will be tracked accurately at different position and shape with help of templates in a considerable amount of time.
\end{abstract}

Keywords: Approximate Nearest neighbour field, Feature Match, Object detection, Gaussian function, Probability density function

\section{Introduction}

Moving object detection in real time application is important challenging task in video surveillance system. There are steps for automatic video analysis. There are object identification, object detection object tracking. As the first step, object identification aims is to identify the object in the region. Then the object detection aims to locate and segment interesting objects in a video. Then, such object can be tracked from frame to frame, and the tracked object can be analysed.

The major application of object detection and tracking are broader monitoring and protection etc. Thus object detection play major role in various field.

Then Background subtraction is commonly used technique for segmentation of motion object in static scenes. It will attempts to detect moving object regions by subtracting the current image that is created by averaging images over time period. Unfortunately the background subtraction model is complex and computationally expensive.

Therefore most of existing approaches for moving object detection and tracking are computationally difficult and subject to more delay and affect the performance of realtime surveillance. 


\section{Related Work}

The literature reveals that a number of approaches have been developed to solve the low level dimension problems that are broadly classified based on the techniques - first order the ANNF computation between source and target image using feature match technique for low dimensional using $\mathrm{k}$ tree algorithm ( $\mathrm{S}$. Avinash Ramakanth and Venkatesh Babu, may 2014).then the ANNF can be used in two application such as super resolution and optical detection. In this computation the optical disk can be detected between source and target image. (S.A.Ramakanth and R.V.Babu, 2014.).p finder is a real time system for tracking people and interpreting their behaviour, it has an ability to find and follow people's head ,hands ,body. Therefore it is an important visual problem (C.Wen, A.Azarbayejani, T.Darrell, and A.Pentland, 1997). This paper has shown a novel, probabilistic method for background subtraction. It involves modelling each pixel as a separate mixture model. These two parameters are robust to different cameras and different scenes. This method deals with slow lighting changes by slowly adapting the values of the Gaussians.(C.Stauffer and Grimson ,"Adaptive background mixture models for real time tracking",1999).using these real-time surveillance the people activities can be detected and tracked usingW4.the W4 is real time visual surveillance system for detecting and tracking the multiple people activities('W4:Real time surveillance of people and their activities',2000).Then tracking the moving object using the techniques background subtraction .using these techniques the object can be detected and also the shadow of the object will be detected(J.Jacques,C.Jung, and S.Musse, "Background subtraction and shadow detection in gray scale video sequences,2005"). Then the object can be tracked spatially using kernel-based tracking ("D.Comaiciu, V.Ramesh, P.Meer", kernel-based object tracking", 2003). A new method for real-time tracking of non-rigid objects seen from a moving camera is proposed. The capability of the tracker to hand le in real-time partial occlusions, signicant clutter, and target scale variations, is demonstrated for several image sequences ("Real-time tracking of non-rigid objects using Mean Shift", 2000). Then based on the colour the images can be retrieved ("colour in image search engines", 2001).Then based on the region the images will be detected, in the sequences of image the region will be selected then the selected region I the object is matched and detected. ("Region tracking through image sequences", 1995).

\section{Overview of the System}

The aim is to build robust and novel moving object detection and tracking algorithm that can detectObject in a variety of challenging real world scenarios. The overall system overview will be represented in diagrammatic form. 


\section{Block Diagram}

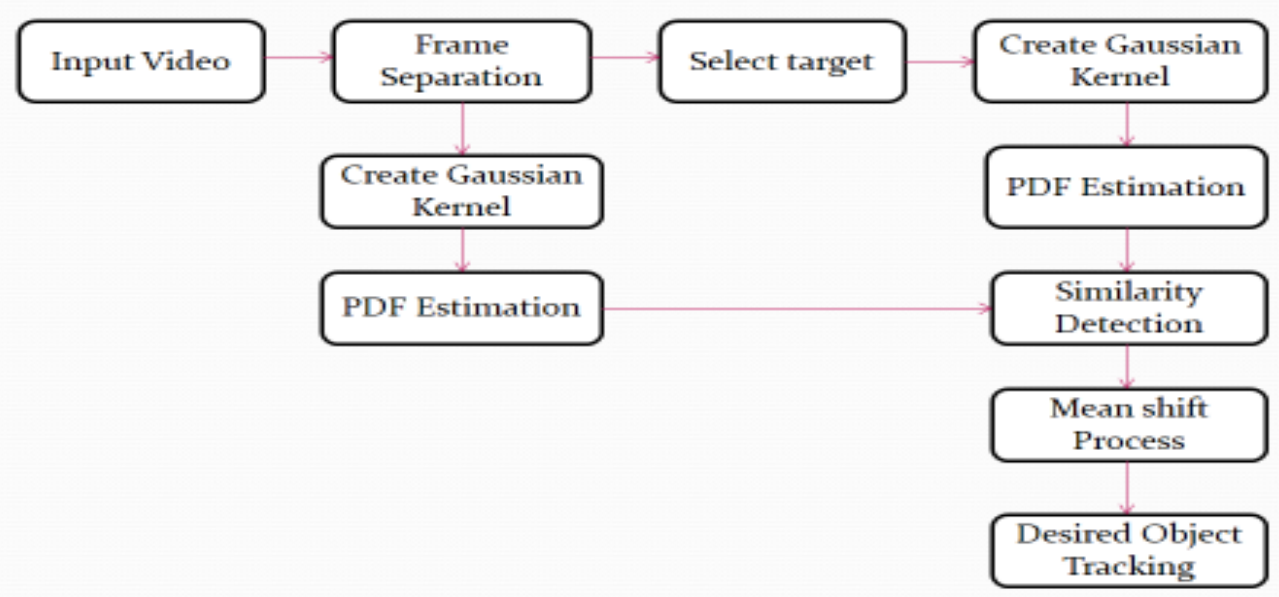

The Steps involved in overall system is

- $\quad$ First process is to select the desired object as a template to detect and track the object in processing frames.

- Template is utilized to estimate the probability density function with Gaussian kernel coefficients.

- Then use the current frames to select the region based on template boundary and find the probability density estimation.

- Measure the similarity between the target PDF and selected regions PDF to locate the object. Then mean shift algorithm is used here to find the desired coordinates in next frames for locating the object.

Finally the object will be tracked and updated based on coordinates evaluated from the mean shift process for next consecutive frames.

\section{Object Detection}

Object detection is initial step in surveillances. There are various algorithm used for detecting the

Object. It will acquire in both static and dynamic background. The challenges like shape, size, occlusion etc. are probably facing.

\subsection{Frame Separation}

An Input Video (.avi files) is converted into still images for processing it and to detect the moving objects. These sequences of images gathered from video files by finding the information about it through 'aviinfo' command. These frames are converted into images with help of the command 'frame2im'. Create the name to each images and this process will be continued for all the video frames. The following diagram represents the process flow of this separation. 


\section{Process Flow}

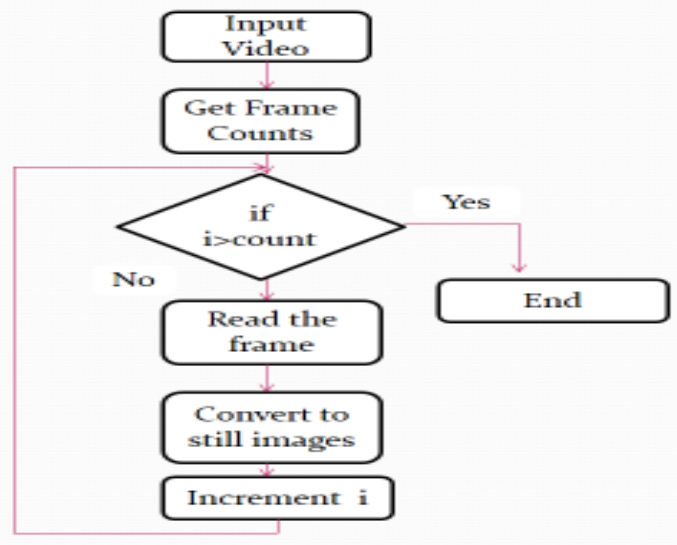

\subsection{Region of Interest}

Region of interest (ROI) is a portion of an image that you want to filter or perform some other operation on. From the source image the region is selected from the input. Then the selected region is compared with selected target image.

\subsection{Gaussian Kernel Function}

Gaussian function are widely used in statistics where they describe the normal distribution, in

Signal processing where they serve to define Gaussian filters, in image processing where two dimensional Gaussian are used for Gaussian blurs, and in mathematics where they are used to solve heat equations and diffusion equations and to define the weirestareas transform. After the region is selected then Gaussian kernel function is defined for filtering. Gaussian process are defined by the joint probability of our observation that joint probability is Gaussian, with mean 0 .

\subsection{PDF Estimation}

The probability density function with Gaussian kernel coefficient is used here to determine the similarity region between target and processing frame. Initially the kernel window will be created using Gaussian function and it is defined by,

$$
\text { Gauss Coeff }=\left(1 / \text { sqrt }\left(2 * \mathrm{pi}^{*} \operatorname{sig}^{\wedge} 2\right)\right)\left(\exp \left(\mathrm{X}^{\wedge} 2+\mathrm{Y}^{\wedge} 2 / 2^{*} \operatorname{sig}^{\wedge} 2\right)\right)
$$

Where $<$ Y, Sig-input coordinates corresponds to the target and Standard Deviation. This estimated PDF will be normalized by created Gaussian kernel reduce the dynamic changes in of an input values.

\subsection{Similarity Detection}

Similarity function is a real value function that quantifies the similarity between two objects. After determining the PDF Estimation the similarity between two images is evaluated. That is the selected target image is compared with source image for detection.

\subsection{Threshold}

The object detection will be performed based on thresholding concept.Thershold is one of the segmentation process. It can determine to segment object and background. It is the process of separating an image into different portions of 
images by selecting a certain grayness level as a threshold and comparing each pixel value with that threshold, and then the threshold valve is compared with similarity, if the threshold value is greater than similarity then the object will be detected otherwise update or reinitialize the region by gradient.

Mean shift based target Updation

- Object detection in next consecutive image will be performed by measuring the similarity between template and input data PDF values.

- The new coordinates belongs to desired target in input from initial one will be determined based on gradient vector obtained from the Gaussian kernel.

- And the coordinates of target dimension will be changed based on this displacement vector, and then new PDF will be estimate to similarity between selected target PDF values.

- This process is continued to get the desired region or up to loss of target during displacement and the gradient vector.

\section{Results and Analysis}

This method was tested on several image sequences. The proposed system was able to detect and track the object in sequences. And it is implemented with the help of MATLAB tool and run on desktop PC with a 3.09GHz Intel and 1.09GB RAM.

In mat lab we have implemented this object tracking and detection algorthim.In this object will be tracked and detected based on mea shift based tacking. Then we evaluated shape, size, color using feature match. The detection output is more accurate and thus can significantly improve the performance of image surveillance based application.

\section{Conclusion and Future Work}

This paper illustrates the suitability of using this model to detect and track the object for surveillance based application. A new technique is applied on the detect object to map its exact on the target image by using its parameter such as size, shape, and color in efficient way with minimum noise.

In a future work, the object detection and tracking present in the scene for more sophisticated vision based application like Navigation, Surveillance, and Medical Field.

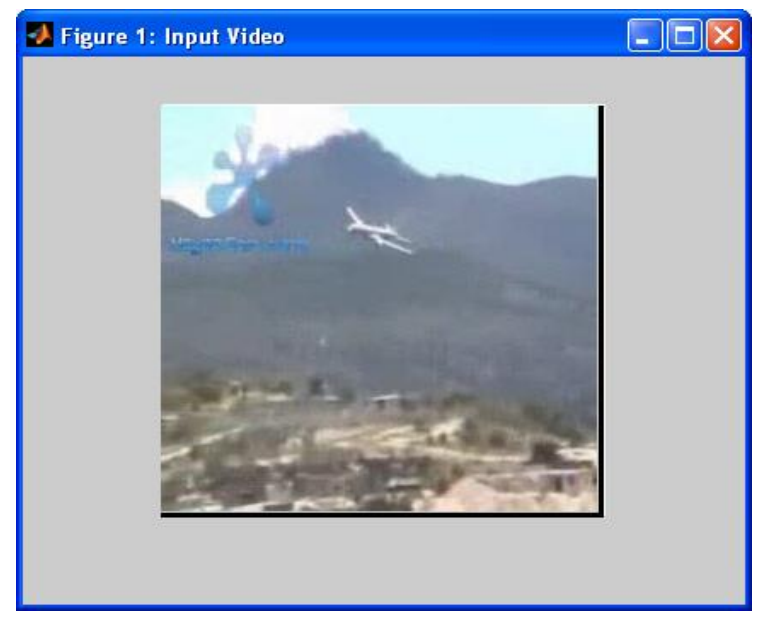




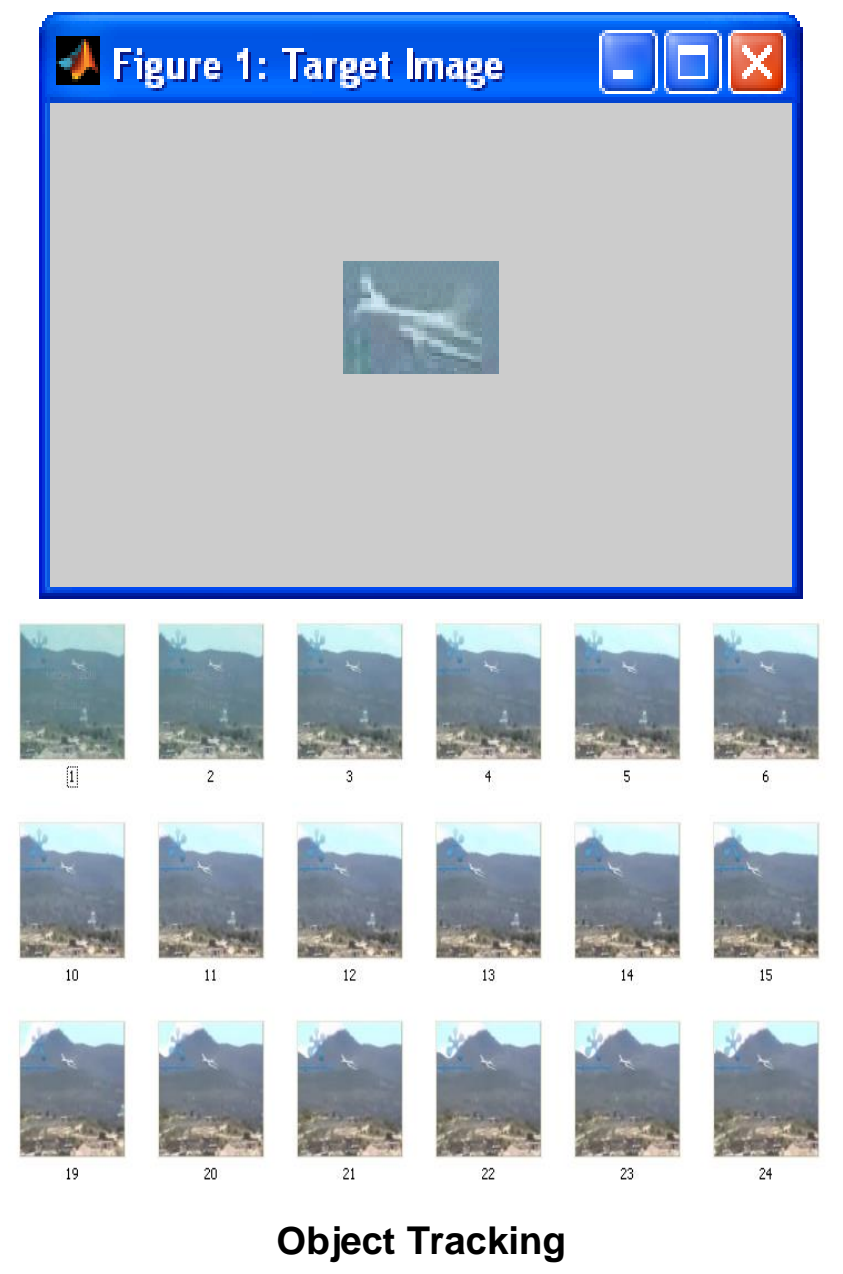

In this moving object detection first the object will be selected in template then the frame has been separated by frame to frame .Then object can be filtered rom the region .then object cab be estimated using density function .then the similarity between the object has determined. When the difference is defined the object will be selected.

Using the Mean Shift tracking the detected object will be tracked from the frame sequences.

\section{Acknowledgment}

I convey my sincere thanks and in depth gratitude to my internal guide Mr.R.Raj Bharath M.E., Assistant

Professor (CSE) of Manakula Vinayagar Institute of Technology for his valuable guidance of my work.

\section{References}

[1] S. A. Ramakanth and R. V. Babu, "Feature Match: A General ANNF Estimation Technique and its Application", vol. 23, no. 5, (2014) May.

[2] S. A. Ramakanth and R. V. Babu, "Approximate nearest neighbour field based optic disk detection, "Comput.Med.Imag.Graph", vol. 38, no. 1, (2014), pp. 49-56.

[3] F. Besse, C. Rother, A. W. Fitzgibbon and J. Kautz, "PMBP: Patch match belief propagation for correspondence field estimation", in Proc. BMVC, (2012), pp. 1-11.9.

[4] S. A. Ramakanth and R. V. Babu, "Feature Match: An efficient low dimensional patch match technique", in Proc. Indian Conf. Comput. Vis., Graph. Image, (2012), pp. 45:1-45:7.

[5] R. F. Sproull, "Refinements to nearest-neighbour searching in k-dimensional trees", Algorithmic a, vol. 6, no. 4, (1991), pp. 579-589. 
[6] A. Yilmaz, O. Javed, and M. Shah, "Object tracking: A survey," ACM Comput. Surv. vol. 38, Dec. 2006

[7] C.Wren, A. Azarbayejani, T. Darrell, and A. Pentland, "Pfinder: Realtime tracking of the human body," IEEE Trans. Patt. Anal. Mach. Intell., vol. 19, no. 7, pp. 780-785, Jul. 1997.

[8] C. Stauffer and W. Grimson, "Adaptive background mixture models for real-time tracking," in IEEE Comput Soc. Conf. CVPR, 1999, pp. 246-252

[9] I. Haritaoglu, D. Harwood, and L. Davis, "W4: Real-time surveillance of people and their activities," IEEE Trans. Patt. Anal. Mach. Intell., vol. 22, no. 8, pp. 809-830, Aug. 2000

[10] J. Jacques, C. Jung, and S. Musse, "Background subtraction and shadow detection in grayscale video sequences," in Eighteenth Brazilian Symp, Computer Graphics and Image Processing, Oct. 2005, pp. 189-196.

[11] D. Comaniciu, V. Ramesh, P. Meer, "Kernel-Based Object Tracking”, IEEE Trans. on Pattern Analysis and Machine Intelligence, vol.25, No. 5, 2003.

[12] D. Comaniciu, V. Ramesh, P. Meer, "Real-Time Tracking of Non-Rigid Objects using Mean Shift", IEEE Conf. on Computer Vision and Pattern Recognition (CVPR '00), vol.2, 2000.10

[13] Th. Gevers, "Color in Image Search Engines", Survey on color for image retrieval from Multimedia Search, ed. M. Lew, Springer Verlag, 2001.

[14] D. Comaniciu and P. Meer, "Mean Shift: A robust approach toward feature space analysis", IEEE Trans. On Pattern Analysis and Machine Intelligence, vol.24, No. 5, 2002

[15] B. Bascle and R. Deriche, "Region tracking through image sequences", in Proc. of 5th Intl. Conference on Computer Vision, Cambridge, MA, 1995.

\section{Authors}

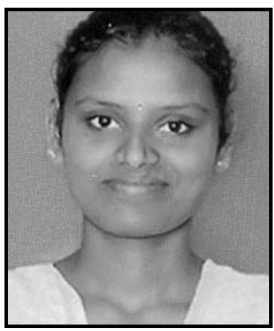

Raj Bharat.R, presently working as Associate Professor in Department of Computer Science and Engineering in Manakula Vinayagar Institute of Engineering and Technology (affiliated to Pondicherry University, Puducherry).

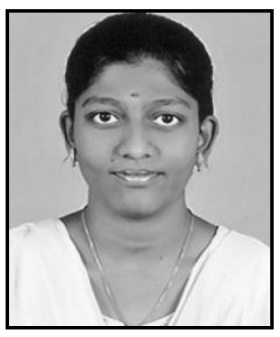

Bavani.P completed Bachelor of degree in Information Technology at 2012 and Pursuing Master degree in Computer Science and Engineering under Pondicherry University, MIT (Manakula Vinayagar Institute of Technology)

Bavya. D, completed Bachelor of degree in Information Technology at 2012 and Pursuing Master degree in Computer Science and Engineering under Pondicherry University, MIT (Manakula Vinayagar Institute of Technology) 
International Journal of Signal Processing, Image Processing and Pattern Recognition Vol.9, No.2 (2016) 